\title{
On weak exponential expansiveness of skew-evolution semiflows in Banach spaces
}

\author{
Tian Yue ${ }^{1,2}$, Xiao-qiu Song ${ }^{1 *}$ and Dong-qing $\mathrm{Li}^{1}$
}

${ }^{*}$ Correspondence: sxqcumt@163.com

${ }^{1}$ College of Sciences, China University of Mining and

Technology, Xuzhou, Jiangsu 221008, China

Full list of author information is available at the end of the article

\begin{abstract}
The aim of this paper is to give several characterizations for weak exponential expansiveness properties of skew-evolution semiflows in Banach spaces. Variants for weak exponential expansiveness of some well-known results in uniform exponential stability theory (Datko (1973)) and exponential instability theory (Lupa (2010), Megan et al. (2008)) are obtained.
\end{abstract}

MSC: Primary 93D20; secondary 34D20

Keywords: skew-evolution semiflow; weak exponential expansiveness; Barreira-Valls weak exponential expansiveness; Lyapunov function

\section{Introduction}

It is well known that in recent years, the exponential stability theory of one parameter semigroups of operators and evolution operators has witnessed significant development. A number of long-standing open problems have been solved and the theory seems to have obtained a certain degree of maturity. One of the most important results of the stability theory is due to Datko, who proved in 1970 in [1] that a strongly continuous semigroup of operators $\{T(t)\}_{t \geq 0}$ is uniformly exponentially stable if and only if for each vector $x$ from the Banach space $X$, the function $t \rightarrow\|T(t) x\|$ lies in $L^{2}\left(\mathbb{R}_{+}\right)$. Later, Pazy generalizes the result in [2] for $L^{p}\left(\mathbb{R}_{+}\right), p \geq 1$. In 1973, Datko [3] generalized the results above, and proved that an evolutionary process $\mathcal{U}=\{U(t, s)\}_{t \geq s \geq 0}$ with uniform exponential growth is uniformly exponentially stable if and only if there exists an exponent $p \geq 1$ such that $\sup _{s \geq 0} \int_{s}^{\infty}\|U(t, s) x\|^{p} d t<\infty$, for each $x \in X$. This result was improved by Rolewicz in 1986 (see [4]). In [5] and [6], the authors generalized the results above in the case of $C_{0^{-}}$ semigroups and evolutionary process, respectively, and presented a unified treatment in terms of Banach function spaces. In [7], the property of nonuniform exponential stability has been studied by L. Barreira and C. Valls. In addition, the weak exponential stability of evolution operators in Banach spaces has been investigated and several important results have been obtained by Lupa, Megan and Popa in [8].

Since the existence problem of exponential expansiveness of evolution equations is distinct compared to the studies devoted to stability and to dichotomy, respectively, exponential expansiveness is a powerful tool when people analyze the asymptotic behavior of dynamical systems. In the last few years, new concepts of exponential expansiveness and in particular, of exponential instability, have been introduced and characterized (see [9-20]). For instance, in [16] Megan and his partners obtained some necessary and sufficient conditions for uniform exponential instability of linear skew-product semiflows in

C2014 Yue et al.; licensee Springer. This is an Open Access article distributed under the terms of the Creative Commons Attribution License (http://creativecommons.org/licenses/by/2.0), which permits unrestricted use, distribution, and reproduction in any medium, provided the original work is properly cited. 
terms of Banach sequence spaces and Banach function spaces. In [12] and [15], the cases of uniform exponential instability has been considered for evolution families and linear skew-product flows, respectively. Additionally, in [19] Lupa considered a weaker notion of instability for evolution operators, thus some necessary and sufficient characterizations for weak exponential instability of evolution operators were obtained.

The concept of skew-evolution semiflows, introduced and characterized by Stoica and Megan in [17] by means of evolution semiflows and cocycles, seems to be more appropriate for the study of asymptotic behaviors of evolution equations. They depend on three variables, contrary to a skew-product semiflow or an evolution operator, for which they are generalizations and which depend only on two. The exponential instability and uniform exponential stability for skew-evolution semiflows are studied by Stoica and Megan in [17] and [21], respectively.

In the present paper, we introduce the concept of weak exponential expansiveness for skew-evolution semiflows which is an extension of classical concept of exponential expansiveness. Our main objective is to give some characterizations for weak exponential expansiveness properties of skew-evolution semiflows in Banach spaces, and variants for weak exponential expansiveness of some well-known results in uniform exponential stability theory (Datko [3]) and exponential instability theory (Lupa [19], Megan and Stoica [17]) are obtained. As applications we obtain characterizations of the concepts in terms of Lyapnov functions. We note that in our proof we don't need to assume the strong continuity of skew-evolution semiflows.

\section{Preliminaries}

Let $(X, d)$ be a metric space, $V$ a Banach spaces. The norm on $V$ and on the space $\mathcal{B}(V)$ of all bounded operators on $V$ will be denoted by $\|\cdot\|$. We denote $T=\left\{\left(t, t_{0}\right) \in \mathbb{R}_{+}^{2}: t \geq t_{0} \geq 0\right\}$ and $Y=X \times V . I$ is the identity operator.

Definition 2.1 (see [21]) A mapping $\varphi: T \times X \rightarrow X$ is called evolution semiflow on $X$ if following properties are satisfied:

(es1) $\varphi(t, t, x)=x, \forall(t, x) \in \mathbb{R}_{+} \times X$;

(es2) $\varphi\left(t, r, \varphi\left(r, t_{0}, x\right)\right)=\varphi\left(t, t_{0}, x\right), \forall(t, r),\left(r, t_{0}\right) \in T, \forall x \in X$

Definition 2.2 (see [21]) A mapping $\Phi: T \times X \rightarrow \mathcal{B}(V)$ is called evolution cocycle over an evolution semiflow $\varphi$ if it satisfies following properties:

(ec1) $\Phi(t, t, x)=I, \forall t \geq 0, \forall x \in X$;

(ec2) $\Phi\left(t, r, \varphi\left(r, t_{0}, x\right)\right) \Phi\left(r, t_{0}, x\right)=\Phi\left(t, t_{0}, x\right), \forall(t, r),\left(r, t_{0}\right) \in T, \forall x \in X$.

Definition 2.3 (see [21]) A mapping $C: T \times Y \rightarrow Y$ defined by

$$
C(t, r, x, v)=(\varphi(t, r, x), \Phi(t, r, x) v)
$$

where $\Phi$ is an evolution cocycle over an evolution semiflow $\varphi$, is called a skew-evolution semiflow on $Y$.

Example 2.4 Let us denote $\mathcal{C}=\mathcal{C}\left(\mathbb{R}_{+}, \mathbb{R}\right)$ the set of all continuous functions $x: \mathbb{R}_{+} \rightarrow \mathbb{R}$, endowed with the topology of uniform convergence on bounded sets. The set $\mathcal{C}$ is metriz- 
able with respect to the metric

$$
d(x, y)=\sum_{n=1}^{\infty} \frac{1}{2^{n}} \frac{d_{n}(x, y)}{1+d_{n}(x, y)}, \quad \text { where } d_{n}(x, y)=\sup _{t \in[-n, n]}|x(t)-y(t)| .
$$

A function $f: \mathbb{R}_{+} \rightarrow[1, \infty)$ defined by $f(t)=e^{-t}+1, t \geq 0$. We denote $f_{t}(\tau)=f(t+\tau)$, $\forall t, \tau \in \mathbb{R}_{+}$. Let $X$ be the closure of the set $\left\{f_{t}, t \in \mathbb{R}_{+}\right\}$in $\mathcal{C}$. Then $(X, d)$ is a metric space and the mapping

$$
\varphi: T \times X \rightarrow X, \quad \varphi(t, r, x)=x_{t-r}
$$

is an evolution semiflow on $X$. Let $V=\mathbb{R}$. We consider $\Phi: T \times X \rightarrow \mathcal{B}(V)$ given by

$$
\Phi(t, r, x) v=e^{\int_{r}^{t} x(\tau-r) d \tau} v,
$$

which is an evolution cocycle and $C=(\varphi, \Phi)$ is a skew-evolution semiflow on $Y$.

Remark 2.5 The skew-evolution semiflows are generalizations of the evolution operators and of the skew-product semiflows (cf. [21], Example 2 and 3).

Definition 2.6 A skew-evolution semiflow $C=(\varphi, \Phi)$ is called with exponential decay if there are $M, \omega>0$ such that

$$
\left\|\Phi\left(t, t_{0}, x_{0}\right) v_{0}\right\| \geq M e^{-\omega(t-r)}\left\|\Phi\left(r, t_{0}, x_{0}\right) v_{0}\right\|,
$$

for all $(t, r),\left(r, t_{0}\right) \in T$ and all $\left(x_{0}, v_{0}\right) \in Y$.

Definition 2.7 A skew-evolution semiflow $C=(\varphi, \Phi)$ is said to be uniformly expansive if there exists a constant $N>0$ such that

$$
\left\|\Phi\left(t, t_{0}, x_{0}\right) v_{0}\right\| \geq N\left\|\Phi\left(r, t_{0}, x_{0}\right) v_{0}\right\|,
$$

for all $(t, r),\left(r, t_{0}\right) \in T$ and all $\left(x_{0}, v_{0}\right) \in Y$.

Definition 2.8 A skew-evolution semiflow $C=(\varphi, \Phi)$ is said to be uniformly exponentially expansive if there are $N, \alpha>0$ such that

$$
\left\|\Phi\left(t, t_{0}, x_{0}\right) v_{0}\right\| \geq N e^{\alpha(t-r)}\left\|\Phi\left(r, t_{0}, x_{0}\right) v_{0}\right\|,
$$

for all $(t, r),\left(r, t_{0}\right) \in T$ and all $\left(x_{0}, v_{0}\right) \in Y$.

Remark 2.9 It is obvious that a skew-evolution semiflow $C=(\varphi, \Phi)$ is uniformly exponentially expansive if and only if there are $N, \alpha>0$ such that

$$
\left\|\Phi\left(t, t_{0}, x_{0}\right) v_{0}\right\| \geq N e^{\alpha\left(t-t_{0}\right)}\left\|v_{0}\right\|
$$

for all $\left(t, t_{0}, x_{0}, v_{0}\right) \in T \times Y$. 
Remark 2.10 If a skew-evolution semiflow is uniformly exponentially expansive then it is uniformly expansive. The converse is not necessarily valid. To show this we consider the following example.

Example 2.11 We consider $X=\mathbb{R}_{+}, V=\mathbb{R}$ and a non-decreasing and bounded function $f: \mathbb{R}_{+} \rightarrow[1, \infty)$. It is obvious that the mapping $\varphi: T \times \mathbb{R}_{+} \rightarrow \mathbb{R}_{+}$defined by

$$
\varphi\left(t, r, x_{0}\right)=t-r+x_{0}, \quad\left(t, r, x_{0}\right) \in T \times \mathbb{R}_{+}
$$

is an evolution semiflow on $\mathbb{R}_{+}$and the mapping $\Phi: T \times \mathbb{R}_{+} \rightarrow \mathcal{B}(\mathbb{R})$ given by

$$
\Phi\left(t, r, x_{0}\right)=\frac{f\left(t-r+x_{0}\right)}{f\left(x_{0}\right)}, \quad\left(t, r, x_{0}\right) \in T \times \mathbb{R}_{+}
$$

is an evolution cocycle on $\mathbb{R}$. Then the skew-evolution semiflow $C=(\varphi, \Phi)$ is uniformly expansive with $N=1$.

On the other hand, if we assume that $C=(\varphi, \Phi)$ is uniformly exponentially expansive then there are constants $N, \alpha>0$ such that

$$
\frac{f\left(t-r+x_{0}\right)}{f\left(x_{0}\right)} \geq N e^{\alpha(t-r)}, \quad \forall\left(t, r, x_{0}\right) \in T \times \mathbb{R}_{+} .
$$

From this, for $r=0$ we obtain $f\left(t+x_{0}\right) \geq N e^{\alpha t} f\left(x_{0}\right)$ which for $t \rightarrow \infty$ gives a contradiction and hence $C$ is not uniformly exponentially expansive.

Definition 2.12 A skew-evolution semiflow $C=(\varphi, \Phi)$ is called weakly exponentially expansive if there are $N, \alpha>0$ such that for all $\left(x_{0}, v_{0}\right) \in Y$ there exists $t_{0} \geq 0$ with

$$
\left\|\Phi\left(t, t_{0}, x_{0}\right) v_{0}\right\| \geq N e^{\alpha(t-r)}\left\|\Phi\left(r, t_{0}, x_{0}\right) v_{0}\right\|
$$

for all $t \geq r \geq t_{0}$.

Remark 2.13 If a skew-evolution semiflow is uniformly exponentially expansive then it is weakly exponentially expansive.

The following example shows that the converse is not valid.

Example 2.14 We consider the metric space $X$ and an evolution semiflow $\varphi$ on $X$ defined as in Example 2.11. Let $V=\mathbb{R}^{2}$ with the Euclidean norm, and the evolution operator $U\left(t, t_{0}\right)=P\left(t, t_{0}\right) Q\left(t_{0}\right)$ (also see [19], Example 11), where

$$
P\left(t, t_{0}\right)=\left(\begin{array}{cc}
e^{t-t_{0}} \sin t & e^{-\left(t-t_{0}\right)} \cos t \\
-e^{t-t_{0}} \cos t & e^{-\left(t-t_{0}\right)} \sin t
\end{array}\right) \quad \text { and } \quad Q\left(t_{0}\right)=\left(\begin{array}{cc}
\cos t_{0} & \sin t_{0} \\
\sin t_{0} & -\cos t_{0}
\end{array}\right) .
$$

Then the mapping $\Phi_{U}: T \times X \rightarrow \mathcal{B}\left(\mathbb{R}^{2}\right)$ given by $\Phi_{U}\left(t, t_{0}, x_{0}\right)=U\left(t, t_{0}\right)$ is an evolution cocycle on $\mathbb{R}^{2}$ over the evolution semiflow $\varphi$.

For every $v_{0} \in \mathbb{R}^{2}$ there exist $\rho \geq 0$ and $t_{0} \in[0,2 \pi)$ such that $v_{0}=\left(\rho \cos t_{0}, \rho \sin t_{0}\right)^{T}$. It is easy to see that

$$
\Phi_{U}\left(t, t_{0}, x_{0}\right) v_{0}=P\left(t, t_{0}\right) Q\left(t_{0}\right) v_{0}=P\left(t, t_{0}\right)(\rho, 0)^{T}=\left(\rho e^{t-t_{0}} \sin t,-\rho e^{t-t_{0}} \cos t\right)^{T}
$$


and hence

$$
\left\|\Phi_{U}\left(t, t_{0}, x_{0}\right) v_{0}\right\|=\rho e^{t-t_{0}}=e^{t-r}\left\|\Phi_{U}\left(r, t_{0}, x_{0}\right) v_{0}\right\|,
$$

for all $t \geq r \geq t_{0}$, which proves that the skew-evolution semiflow $C=(\varphi, \Phi)$ is weakly exponentially expansive.

On the other hand, we observe that for $y_{0}=\left(\sin t_{0},-\cos t_{0}\right)^{T}$,

$$
\Phi_{U}\left(t, t_{0}, x_{0}\right) y_{0}=P\left(t, t_{0}\right)(0,1)^{T}=\left(e^{-\left(t-t_{0}\right)} \cos t, e^{-\left(t-t_{0}\right)} \sin t\right)^{T}
$$

and hence

$$
\left\|\Phi_{U}\left(t, t_{0}, x_{0}\right) y_{0}\right\|=e^{-\left(t-t_{0}\right)}=e^{-(t-r)}\left\|\Phi_{U}\left(r, t_{0}, x_{0}\right) y_{0}\right\|,
$$

which shows that $C$ is not uniformly exponentially expansive.

Definition 2.15 A skew-evolution semiflow $C=(\varphi, \Phi)$ is called weakly exponentially expansive in the Barreira-Valls sense if there are $N, \alpha>0$ and $\beta \geq 0$ such that for all $\left(x_{0}, v_{0}\right) \in Y$ there exists $t_{0} \geq 0$ with

$$
e^{\beta r}\left\|\Phi\left(t, t_{0}, x_{0}\right) v_{0}\right\| \geq N e^{\alpha(t-r)}\left\|\Phi\left(r, t_{0}, x_{0}\right) v_{0}\right\|
$$

for all $t \geq r \geq t_{0}$.

Definition 2.16 (see [17]) A skew-evolution semiflow $C=(\varphi, \Phi)$ is called strongly measurable if the mapping $t \mapsto\left\|\Phi\left(t, t_{0}, x_{0}\right) v_{0}\right\|$ is measurable on $\left[t_{0}, \infty\right)$ for all $\left(t_{0}, x_{0}, v_{0}\right) \in$ $\mathbb{R}_{+} \times Y$.

Definition 2.17 A mapping $\mathcal{L}: T \times Y \rightarrow \mathbb{R}$ is said to be a Lyapunov function for the skewevolution semiflow $C=(\varphi, \Phi)$ if there is a constant $a \geq 0$ such that for all $\left(x_{0}, v_{0}\right) \in Y$ there exists $t_{0} \geq 0$ with

$$
\mathcal{L}\left(t, t_{0}, x_{0}, v_{0}\right)+\int_{r}^{t} e^{a(t-s)}\left\|\Phi\left(s, t_{0}, x_{0}\right) v_{0}\right\|^{2} d s \leq \mathcal{L}\left(r, t_{0}, x_{0}, v_{0}\right)
$$

for all $t \geq r \geq t_{0}$.

\section{The main results}

Proposition 3.1 A skew-evolution semiflow $C=(\varphi, \Phi)$ is weakly exponentially expansive if and only if there exists a decreasing function $f:[0, \infty) \rightarrow(0, \infty)$ with $\lim _{t \rightarrow \infty} f(t)=0$ such that for every $\left(x_{0}, v_{0}\right) \in Y$ there is $t_{0} \geq 0$ with the property

$$
\left\|\Phi\left(r, t_{0}, x_{0}\right) v_{0}\right\| \leq f(t-r)\left\|\Phi\left(t, t_{0}, x_{0}\right) v_{0}\right\|,
$$

for all $t \geq r \geq t_{0}$. 
Proof Necessity. It is a simple verification for $f(t)=\frac{1}{N} e^{-\alpha t}$, where $N$ and $\alpha$ are given by Definition 2.12.

Sufficiency. According to the property of function $f$, there exists a constant $\delta>0$ such that $f(\delta)<1$. From the hypothesis we find that for every $\left(x_{0}, v_{0}\right) \in Y$ there is $t_{0} \geq 0$ satisfying relation (8). For every $t \geq r \geq t_{0}$ there are $n \in \mathbb{N}$ and $l \in[0, \delta)$ such that $t-r=n \delta+l$. Then the following inequalities:

$$
\begin{aligned}
\left\|\Phi\left(r, t_{0}, x_{0}\right) v_{0}\right\| & \leq f(l)\left\|\Phi\left(t-n \delta, t_{0}, x_{0}\right) v_{0}\right\| \\
& \leq f(l) f(\delta)\left\|\Phi\left(t-(n-1) \delta, t_{0}, x_{0}\right) v_{0}\right\| \\
& \leq \cdots \\
& \leq f(l)[f(\delta)]^{n}\left\|\Phi\left(t, t_{0}, x_{0}\right) v_{0}\right\| \\
& \leq \frac{f(0)}{f(\delta)}[f(\delta)]^{n+1}\left\|\Phi\left(t, t_{0}, x_{0}\right) v_{0}\right\| \\
& \leq \frac{1}{N} e^{-\alpha(t-r)}\left\|\Phi\left(t, t_{0}, x_{0}\right) v_{0}\right\|
\end{aligned}
$$

hold for all $t \geq r \geq t_{0}$, where we have denoted $N=\frac{f(\delta)}{f(0)}$ and $\alpha=-\frac{\ln f(\delta)}{\delta}$.

Finally, it follows that $C=(\varphi, \Phi)$ is weakly exponentially expansive.

Corollary 3.2 A skew-evolution semiflow $C=(\varphi, \Phi)$ is weakly exponentially expansive if and only if there exists a non-decreasing function $g:[0, \infty) \rightarrow(0, \infty)$ with $\lim _{t \rightarrow \infty} g(t)=$ $+\infty$ such that for every $\left(x_{0}, v_{0}\right) \in Y$ there is $t_{0} \geq 0$ with the property

$$
g(t-r)\left\|\Phi\left(r, t_{0}, x_{0}\right) v_{0}\right\| \leq\left\|\Phi\left(t, t_{0}, x_{0}\right) v_{0}\right\|
$$

for all $t \geq r \geq t_{0}$.

Theorem 3.3 Let $C=(\varphi, \Phi)$ be a strongly measurable skew-evolution semiflow with exponential decay. Then $C$ is weakly exponentially expansive if and only if there are $p>0$ and $L>0$ such that for every $\left(x_{0}, v_{0}\right) \in Y$ there is $t_{0} \geq 0$ with

$$
\int_{t_{0}}^{t}\left\|\Phi\left(s, t_{0}, x_{0}\right) v_{0}\right\|^{p} d s \leq L\left\|\Phi\left(t, t_{0}, x_{0}\right) v_{0}\right\|^{p}
$$

for all $t \geq t_{0}$.

Proof Necessity. If $C$ is weakly exponentially expansive then from Definition 2.12 it follows that there are $N, \alpha>0$ with the property that for all $\left(x_{0}, v_{0}\right) \in Y$ there exists $t_{0} \geq 0$ such that

$$
\begin{aligned}
& \int_{t_{0}}^{t}\left\|\Phi\left(s, t_{0}, x_{0}\right) v_{0}\right\|^{p} d s \\
& \quad \leq N^{-p} \int_{t_{0}}^{t} e^{-\alpha p(t-s)} d s\left\|\Phi\left(t, t_{0}, x_{0}\right) v_{0}\right\|^{p} \\
& \quad \leq L\left\|\Phi\left(t, t_{0}, x_{0}\right) v_{0}\right\|^{p}
\end{aligned}
$$

for all $t \geq t_{0}$, where $p>0$ is fixed and $L=\frac{N^{-p}}{\alpha p}$. 
Sufficiency. We assume that there are $p>0$ and $L>0$ such that for every $\left(x_{0}, v_{0}\right) \in Y$ there is $t_{0} \geq 0$ satisfying inequality (10). Let $t \geq r \geq t_{0}$. If $t \geq r+1$ we have

$$
\begin{aligned}
L\left\|\Phi\left(t, t_{0}, x_{0}\right) v_{0}\right\|^{p} & \geq \int_{t_{0}}^{t}\left\|\Phi\left(s, t_{0}, x_{0}\right) v_{0}\right\|^{p} d s \geq \int_{r}^{t}\left\|\Phi\left(s, t_{0}, x_{0}\right) v_{0}\right\|^{p} d s \\
& \geq M^{p} \int_{r}^{t} e^{-\omega p(s-r)} d s\left\|\Phi\left(r, t_{0}, x_{0}\right) v_{0}\right\|^{p} \\
& =M^{p} \int_{0}^{t-r} e^{-\omega p \tau} d \tau\left\|\Phi\left(r, t_{0}, x_{0}\right) v_{0}\right\|^{p} \\
& \geq M^{p} \int_{0}^{1} e^{-\omega p \tau} d \tau\left\|\Phi\left(r, t_{0}, x_{0}\right) v_{0}\right\|^{p} \\
& =\frac{1-e^{-p \omega}}{p \omega} M^{p}\left\|\Phi\left(r, t_{0}, x_{0}\right) v_{0}\right\|^{p}
\end{aligned}
$$

and for $t \in[r, r+1)$ we have

$$
\left\|\Phi\left(t, t_{0}, x_{0}\right) v_{0}\right\|^{p} \geq e^{-p \omega} M^{p}\left\|\Phi\left(r, t_{0}, x_{0}\right) v_{0}\right\|^{p},
$$

where $M, \omega>0$ are given by Definition 2.6.

Hence

$$
\left\|\Phi\left(t, t_{0}, x_{0}\right) v_{0}\right\|^{p} \geq K\left\|\Phi\left(r, t_{0}, x_{0}\right) v_{0}\right\|^{p}
$$

for all $t \geq r \geq t_{0}$, where $K=M^{p}\left[e^{-p \omega}+\left(1-e^{-p \omega}\right) / p \omega L\right]$.

On the other hand

$$
\begin{aligned}
L\left\|\Phi\left(t, t_{0}, x_{0}\right) v_{0}\right\|^{p} & \geq \int_{t_{0}}^{t}\left\|\Phi\left(s, t_{0}, x_{0}\right) v_{0}\right\|^{p} d s \geq \int_{r}^{t}\left\|\Phi\left(s, t_{0}, x_{0}\right) v_{0}\right\|^{p} d s \\
& \geq K(t-r)\left\|\Phi\left(r, t_{0}, x_{0}\right) v_{0}\right\|^{p},
\end{aligned}
$$

for all $t \geq r \geq t_{0}$.

Adding up (11) and (12) we obtain

$$
\left(1+L^{1 / p}\right)\left\|\Phi\left(t, t_{0}, x_{0}\right) v_{0}\right\| \geq K^{1 / p}\left[1+(t-r)^{1 / p}\right]\left\|\Phi\left(r, t_{0}, x_{0}\right) v_{0}\right\|,
$$

for all $t \geq r \geq t_{0}$. According to Corollary 3.2, $C$ is weakly exponentially expansive, which ends the proof.

Theorem 3.4 Let $C=(\varphi, \Phi)$ be a strongly measurable skew-evolution semiflow with exponential decay. Then $C$ is weakly exponentially expansive if and only if there are $\mathcal{L}: T \times Y \rightarrow$ $\mathbb{R}_{-}$a Lyapunov function for $C$ and $a$ constant $b>0$ such that

$$
\left|\mathcal{L}\left(t, t_{0}, x_{0}, v_{0}\right)\right| \leq b\left\|\Phi\left(t, t_{0}, x_{0}\right) v_{0}\right\|^{2},
$$

for all $t \geq t_{0}$. 
Proof Necessity. Let $a=0$. We consider the application $\mathcal{L}: T \times Y \rightarrow \mathbb{R}_{-}$,

$$
\mathcal{L}(t, r, x, v)=-\int_{r}^{t} e^{a(t-s)}\|\Phi(s, r, x) v\|^{2} d s \leq 0 .
$$

Then from Definition 2.12 we find that there are $N, \alpha>0$, and for every $\left(x_{0}, v_{0}\right) \in Y$ there is $t_{0} \geq 0$ with

$$
\left|\mathcal{L}\left(t, t_{0}, x_{0}, v_{0}\right)\right|=\int_{t_{0}}^{t}\left\|\Phi\left(s, t_{0}, x_{0}\right) v_{0}\right\|^{2} d s \leq b\left\|\Phi\left(t, t_{0}, x_{0}\right) v_{0}\right\|^{2},
$$

for all $t \geq t_{0}$, where $b=\frac{1}{2 \alpha N^{2}}$. It is easy to see that

$$
\mathcal{L}\left(t, t_{0}, x_{0}, v_{0}\right)+\int_{r}^{t} e^{a(t-s)}\left\|\Phi\left(s, t_{0}, x_{0}\right) v_{0}\right\|^{2} d s-\mathcal{L}\left(r, t_{0}, x_{0}, v_{0}\right) \leq 0,
$$

for all $t \geq r \geq t_{0}$. Hence $\mathcal{L}$ is a Lyapunov function for $C$ such that the relation (13) is true.

Sufficiency. We assume that there are $\mathcal{L}: T \times Y \rightarrow \mathbb{R}_{-}$a Lyapunov function for $C$ and a constant $b>0$ such that the relation (13) hold.

Then

$$
\begin{aligned}
\int_{t_{0}}^{t}\left\|\Phi\left(s, t_{0}, x_{0}\right) v_{0}\right\|^{2} d s & \leq \int_{t_{0}}^{t} e^{a(t-s)}\left\|\Phi\left(s, t_{0}, x_{0}\right) v_{0}\right\|^{2} d s \\
& \leq \mathcal{L}\left(t_{0}, t_{0}, x_{0}, v_{0}\right)-\mathcal{L}\left(t, t_{0}, x_{0}, v_{0}\right) \\
& \leq-\mathcal{L}\left(t, t_{0}, x_{0}, v_{0}\right)=\left|\mathcal{L}\left(t, t_{0}, x_{0}, v_{0}\right)\right| \\
& \leq b\left\|\Phi\left(t, t_{0}, x_{0}\right) v_{0}\right\|^{2}
\end{aligned}
$$

for all $t \geq t_{0}$, where $a \geq 0$ is given by Definition 2.17. By Theorem 3.3 we conclude that $C$ is weakly exponentially expansive.

Proposition 3.5 A skew-evolution semiflow $C=(\varphi, \Phi)$ is weakly exponentially expansive in the Barreira-Valls sense if and only if there are $N>0, \lambda>\mu \geq 0$ such that for all $\left(x_{0}, v_{0}\right) \in$ $Y$ there exists $t_{0} \geq 0$ with

$$
e^{\mu t}\left\|\Phi\left(t, t_{0}, x_{0}\right) v_{0}\right\| \geq N e^{\lambda(t-r)}\left\|\Phi\left(r, t_{0}, x_{0}\right) v_{0}\right\|
$$

for all $t \geq r \geq t_{0}$.

Proof Necessity. It follows by a simple verification for $\mu=\beta$ and $\lambda=\alpha+\beta$, where constants $\alpha>0$ and $\beta \geq 0$ are given by Definition 2.15 .

Sufficiency. From the hypothesis, there are $N>0, \lambda>\mu \geq 0$ such that for all $\left(x_{0}, v_{0}\right) \in Y$ there exists $t_{0} \geq 0$ satisfying

$$
\begin{aligned}
N\left\|\Phi\left(r, t_{0}, x_{0}\right) v_{0}\right\| & \leq e^{\mu t} e^{-\lambda(t-r)}\left\|\Phi\left(t, t_{0}, x_{0}\right) v_{0}\right\| \\
& =e^{\mu r} e^{-(\lambda-\mu)(t-r)}\left\|\Phi\left(t, t_{0}, x_{0}\right) v_{0}\right\|,
\end{aligned}
$$

for all $t \geq r \geq t_{0}$, which implies that $C$ is weakly exponentially expansive in the BarreiraValls sense with $\alpha=\lambda-\mu$ and $\beta=\mu$. 
Theorem 3.6 Let $C=(\varphi, \Phi)$ be a strongly measurable skew-evolution semiflow with exponential decay. Then $C$ is weakly exponentially expansive in the Barreira-Valls sense if and only if there are $L, \alpha>0, p>0$ and $\beta \geq 0$ such that for every $\left(x_{0}, v_{0}\right) \in Y$ there is $t_{0} \geq 0$ with

$$
\int_{t_{0}}^{t} e^{p(\alpha+\beta)(t-s)}\left\|\Phi\left(s, t_{0}, x_{0}\right) v_{0}\right\|^{p} d s \leq L e^{p \beta t}\left\|\Phi\left(t, t_{0}, x_{0}\right) v_{0}\right\|^{p},
$$

for all $t \geq t_{0}$.

Proof Necessity. If $C$ is weakly exponentially expansive in the Barreira-Valls sense, then by Proposition 3.5 there are $N>0, \lambda>\mu \geq 0$ such that for all $\left(x_{0}, v_{0}\right) \in Y$ there exists $t_{0} \geq 0$ with

$$
\begin{aligned}
& \int_{t_{0}}^{t} e^{p(\alpha+\beta)(t-s)}\left\|\Phi\left(s, t_{0}, x_{0}\right) v_{0}\right\|^{p} d s \\
& \quad \leq N^{-p} e^{p \mu t} \int_{t_{0}}^{t} e^{-(\lambda-\alpha-\beta) p(t-s)} d s\left\|\Phi\left(t, t_{0}, x_{0}\right) v_{0}\right\|^{p} \\
& \leq L e^{p \beta t}\left\|\Phi\left(t, t_{0}, x_{0}\right) v_{0}\right\|^{p},
\end{aligned}
$$

for all $t \geq r \geq t_{0}$, where $p>0$ is fixed, $\beta=\mu, \alpha \in(0, \lambda-\mu)$ and $L=\frac{N^{-p}}{p(\lambda-\alpha-\beta)}$.

Sufficiency. We assume that there are $L, \alpha>0, p>0$ and $\beta \geq 0$ such that for every $\left(x_{0}, v_{0}\right) \in Y$ there is $t_{0} \geq 0$ satisfying inequality (15). Let $t \geq r \geq t_{0}$. If $t \geq r+1$ then

$$
\begin{aligned}
& M^{p} e^{-p(\alpha+\beta+\omega)} e^{p(\alpha+\beta)(t-r)}\left\|\Phi\left(r, t_{0}, x_{0}\right) v_{0}\right\|^{p} \\
& \quad=\int_{r}^{r+1} M^{p} e^{-p(\alpha+\beta+\omega)} e^{p(\alpha+\beta)(t-r)}\left\|\Phi\left(r, t_{0}, x_{0}\right) v_{0}\right\|^{p} d s \\
& \quad \leq \int_{r}^{r+1} e^{-p(\alpha+\beta+\omega)} e^{p(s-r)} e^{p(\alpha+\beta)(t-s)} e^{p(\alpha+\beta)(s-r)}\left\|\Phi\left(s, t_{0}, x_{0}\right) v_{0}\right\|^{p} d s \\
& \quad=\int_{r}^{r+1} e^{p(\alpha+\beta+\omega)[(s-r)-1]} e^{p(\alpha+\beta)(t-s)}\left\|\Phi\left(s, t_{0}, x_{0}\right) v_{0}\right\|^{p} d s \\
& \leq \int_{r}^{r+1} e^{p(\alpha+\beta)(t-s)}\left\|\Phi\left(s, t_{0}, x_{0}\right) v_{0}\right\|^{p} d s \\
& \quad \leq L e^{p \beta t}\left\|\Phi\left(t, t_{0}, x_{0}\right) v_{0}\right\|^{p},
\end{aligned}
$$

and therefore

$$
e^{(\alpha+\beta) r}\left\|\Phi\left(t, t_{0}, x_{0}\right) v_{0}\right\| \geq M L^{-1 / p} e^{-(\alpha+\beta+\omega)} e^{\alpha t}\left\|\Phi\left(r, t_{0}, x_{0}\right) v_{0}\right\|
$$

where $M, \omega>0$ are given by Definition 2.6.

We consider $t \in[r, r+1)$. Then

$$
\begin{aligned}
\left\|\Phi\left(r, t_{0}, x_{0}\right) v_{0}\right\| & \leq M^{-1} e^{\omega(t-r)}\left\|\Phi\left(t, t_{0}, x_{0}\right) v_{0}\right\| \\
& =M^{-1} e^{(\alpha+\beta+\omega)(t-r)} e^{-(\alpha+\beta)(t-r)}\left\|\Phi\left(t, t_{0}, x_{0}\right) v_{0}\right\| \\
& \leq M^{-1} e^{(\alpha+\beta+\omega)} e^{-(\alpha+\beta)(t-r)}\left\|\Phi\left(t, t_{0}, x_{0}\right) v_{0}\right\| .
\end{aligned}
$$


Further we obtain

$$
\begin{aligned}
e^{(\alpha+\beta) r}\left\|\Phi\left(t, t_{0}, x_{0}\right) v_{0}\right\| & \geq M e^{-(\alpha+\beta+\omega)} e^{(\alpha+\beta) t}\left\|\Phi\left(r, t_{0}, x_{0}\right) v_{0}\right\| \\
& \geq M e^{-(\alpha+\beta+\omega)} e^{\alpha t}\left\|\Phi\left(r, t_{0}, x_{0}\right) v_{0}\right\| .
\end{aligned}
$$

Hence, $C$ is weakly exponentially expansive in the Barreira-Valls sense, which ends the proof.

Remark 3.7 Theorems 3.3 and 3.6 are the versions of the classical stability theorems and exponential instability theorems due to Datko [3], Lupa [19], Megan and Stoica [17], for weak exponential expansiveness of skew-evolution semiflows.

Theorem 3.8 Let $C=(\varphi, \Phi)$ be a strongly measurable skew-evolution semiflow with exponential decay. Then $C$ is weakly exponentially expansive in the Barreira-Valls sense if and only if there are $\mathcal{L}: T \times Y \rightarrow \mathbb{R}_{-}$a Lyapunov function for $C$ and constants $c>0, d \geq 0$ such that

$$
\left|\mathcal{L}\left(t, t_{0}, x_{0}, v_{0}\right)\right| \leq c e^{d t}\left\|\Phi\left(t, t_{0}, x_{0}\right) v_{0}\right\|^{2}
$$

for all $t \geq t_{0}$.

Proof According to the conclusion of Theorem 3.6, the argumentation can be obtained as well as that of Theorem 3.4.

\section{Competing interests}

The authors declare that they have no competing interests.

Authors' contributions

The authors completed the paper together. They also read and approved the final manuscript.

\section{Author details}

${ }^{1}$ College of Sciences, China University of Mining and Technology, Xuzhou, Jiangsu 221008, China. ${ }^{2}$ School of Science, Hubei University of Automotive Technology, Shiyan, Hubei 442002, China.

\section{Acknowledgements}

The authors would like to thank the referee for helpful suggestions and comments. This work was supported by 'the Fundamental Research Funds for the Central Universities' (No.2013XK03).

Received: 5 June 2013 Accepted: 1 April 2014 Published: 06 May 2014

\section{References}

1. Datko, R: Extending a theorem of Liapunov to Hilbert spaces. J. Math. Anal. Appl. 32, 610-616 (1970)

2. Pazy, A: Semigroups of Linear Operators and Applications to Partial Differential Equations. Springer, New York (1983)

3. Datko, R: Uniform asymptotic stability of evolutionary processes in a Banach space. SIAM J. Math. Anal. 3, 428-445 (1973)

4. Rolewicz, S: On uniform N-equistability. J. Math. Anal. Appl. 115, 434-441 (1986)

5. Neerven, JMAM: Exponential stability of operators and semigroups. J. Funct. Anal. 130, 293-309 (1995)

6. Preda, P, Pogan, A, Preda, C: Functionals on function and sequence spaces connected with the exponential stability of evolutionary processes. Czechoslov. Math. J. 131, 425-435 (2006)

7. Barreira, L, Valls, C: Stability of Nonautonomous Differential Equations. Springer, Berlin (2008)

8. Lupa, N, Megan, M, Popa, I-L: On weak exponential stability of evolution operators in Banach spaces. Nonlinear Anal. 73, 2445-2450 (2010)

9. Minh, NV, Räbiger, F, Schnaubelt, R: Exponential stability, exponential expansiveness and exponential dichotomy of evolution equations on the half-line. Integral Equ. Oper. Theory 32, 332-353 (1998)

10. Megan, M, Sasu, AL, Sasu, B: Nonuniform exponential unstability of evolution operators in Banach spaces. Glas. Mat. Ser. III 36, 287-295 (2001) 
11. Megan, M, Pogan, A: On exponential $h$-expansiveness of semigroups of operators in Banach spaces. Nonlinear Anal. 52, 545-556 (2003)

12. Megan, M, Sasu, AL, Sasu, B: Banach function spaces and exponential instability of evolution families. Arch. Math. 39, 277-286 (2003)

13. Megan, M, Sasu, AL, Sasu, B: Perron conditions for uniform exponential expansiveness of linear skew-product flows. Monatshefte Math. 138, 145-157 (2003)

14. Megan, M, Sasu, B, Sasu, AL: Exponential expansiveness and complete admissibility for evolution families. Czechoslov. Math. J. 54, 1485-1493 (2004)

15. Megan, M, Sasu, AL, Sasu, B: Exponential stability and exponential instability for linear skew-product flows. Math. Bohem. 129, 225-243 (2004)

16. Megan, M, Sasu, AL, Sasu, B: Exponential instability of linear skew-product semiflows in terms of Banach function spaces. Results Math. 45, 309-318 (2004)

17. Megan, M, Stoica, C: Exponential instability of skew-evolution semiflows in Banach spaces. Stud. Univ. "Babeş-Bolyai" Math. LIII, 17-24 (2008)

18. Sasu, B: New criteria for exponential expansiveness of variational difference equations. J. Math. Anal. Appl. 327 287-297 (2007)

19. Lupa, N: Necessary and sufficient conditions for weak exponential instability of evolution operators. Int. J. Pure Appl. Math. 62, 263-273 (2010)

20. Lupa, N, Megan, M: Exponential dichotomies of evolution operators in Banach spaces. Monatshefte Math. (2013). doi:10.1007/s00605-013-0517-y

21. Stoica, C, Megan, M: On uniform exponential stability for skew-evolution semiflows on Banach spaces. Nonlinear Anal. 72, 1305-1313 (2010)

10.1186/1029-242X-2014-165

Cite this article as: Yue et al.: On weak exponential expansiveness of skew-evolution semiflows in Banach spaces. Journal of Inequalities and Applications 2014, 2014:165

\section{Submit your manuscript to a SpringerOpen ${ }^{\circ}$ journal and benefit from:}

- Convenient online submission

- Rigorous peer review

Immediate publication on acceptance

Open access: articles freely available online

High visibility within the field

- Retaining the copyright to your article 